\title{
Biofilm Accumulation and Sucrose Rinse Modulate the Calcium and Fluoride Bioavailability in the Saliva of Children with Early Childhood Caries
}

Camila Lopes Crescente

State University of Campinas

Emerson Tavares de Sousa

State University of Campinas

Aline Tavares Lima-Holanda

State University of Campinas

Carolina Steiner-Oliveira

State University of Campinas

Marinês Nobre-dos-Santos ( $\nabla$ nobre@fop.unicamp.br)

State University of Campinas

\section{Research Article}

Keywords: Dental Caries, Preschoolers, Saliva, Calcium, Phosphate, Fluoride.

Posted Date: January 3rd, 2022

DOI: https://doi.org/10.21203/rs.3.rs-1157133/v1

License: @) (i) This work is licensed under a Creative Commons Attribution 4.0 International License. Read Full License 


\section{Abstract}

This quasi-experimental study aimed at investigating the combined effect of biofilm accumulation and $20 \%$ sucrose rinse on the modulation of calcium $\left(\mathrm{Ca}^{2+}\right)$, phosphate $(\mathrm{Pi})$, and fluoride (F-) bioavailability in saliva of children with early childhood caries (ECC). In-that, fifty-six preschoolers of both genders were evaluated according to caries experience and activity: caries-free $(C F, n=28)$ and with ECC $(n=28)$ and then, submitted to biofilm intervention (biofilm accumulation or no biofilm accumulation). In each situation, saliva samples were collected before and five minutes after a $20 \%$ sucrose rinse to determine the concentrations of $\mathrm{Ca}^{2+}, \mathrm{Pi}$, and $\mathrm{F}^{-}$. Calcium concentration was significantly lower in the biofilm accumulation situation compared to the situation of biofilm mechanical control, except for children CF after sucrose rinse. Biofilm accumulation increased salivary calcium concentration in children with ECC after sucrose rinse, whereas mechanical biofilm control reduced it in both groups. The phosphate concentration was influenced by mechanical control of the biofilm in CF children. The fluoride bioavailability was reduced by sucrose rinse and biofilm accumulation in CF and ECC children. In conclusion, the combined effect of biofilm accumulation and sucrose rinse modifies the bioavailability of calcium and fluoride in the saliva of children with early childhood caries.

\section{Introduction}

Early childhood caries (ECC) is one of the most prevalent chronic diseases in children worldwide (Tinanoff et al., 2019). One of the main factors for the onset and progression of tooth decay is the presence of biofilm, which is significantly impaired by the sugar bioavailability in the oral environment (Koo et al., 2013). Dental biofilm is an organized structure composed of commensal bacteria surrounded by an extracellular matrix that accumulates over the dental structure due to poor oral hygiene (Marsh, 2018). The biofilm function is determined by the composition and properties of saliva, as well as by the dominant nutritional source in the child's diet. In this sense, a diet rich in the consumption of fermentable carbohydrates such as sucrose provides a coercive environment for a highly specialized oral ecosystem with a predominance of saccharolytic species, with important changes in the physicochemical properties of saliva (Zaura et al., 2017).

Saliva plays an important role in maintaining the integrity of dental tissues due to the presence of $\mathrm{Ca}^{2}+, \mathrm{Pi}$, and $\mathrm{F}^{-}$ and other inorganic ions. These chemical components can facilitate the remineralization of early caries lesions (Featherstone, 2004; Pappa et al., 2019) and promote a supersaturation of minerals, acting strategically to mitigate the damage caused by saccharolytic bacteria (Lenander-Lumikari e Loimaranta, 2000; Jain et al., 2018). Thus, $\mathrm{Ca}^{2}+$, $\mathrm{Pi}$, and $\mathrm{F}^{-}$levels in saliva form a natural defense mechanism against tooth enamel dissolution (García-Godoy e Hicks, 2008; Dawes, 2008).

As early demonstrated by de Sousa et al., (2020), sucrose rinse has a significant effect on the electrolytic concentration of $\mathrm{Pi}$ and $\mathrm{F}^{-}$in saliva when children with ECC were compared with CF children. In addition, LimaHolanda et al. (2021) evidenced that the saliva's ability to neutralize the oral environment was different when children with ECC were compared with CF children and that this saliva potential was influenced by both sucrose exposure and biofilm accumulation. Probably, this result may reflect the transient shift of the oral ecosystem after sucrose exposure and can be more deleterious for $\mathrm{pH}$-modifying ability in children with ECC. In this context, considering the influence of saliva $\mathrm{pH}$ and buffering capacity on the electrolytic dynamic in the oral cavity, it was hypothesized that biochemical changes in saliva can be expected as consequence of the cariogenic challenge and biofilm accumulation. 
Therefore, it is pertinent to investigate the changes occurring in the bioavailability of salivary ions such as $\mathrm{Ca}^{2}+, \mathrm{Pi}$, and $\mathrm{F}^{-}$in children with ECC after a sucrose exposure, considering situations of adequate oral hygiene and poor oral hygiene. Thus, this study aimed to investigate the combined effect of biofilm accumulation and sucrose rinse on the modulation of $\mathrm{Ca}^{2}+, \mathrm{Pi}$, and $\mathrm{F}^{-}$bioavailability in the saliva of children with ECC. Our research hypothesis was that in children with ECC, after a sucrose rinse, the biofilm accumulation would modify the bioavailability of $\mathrm{Ca}^{2}+, \mathrm{Pi}$, and $\mathrm{F}^{-}$ minerals differently from caries-free children. Of note, this paper is an extension of a published study (Lima-Holanda et al., 2021).

\section{Results}

Fifty-six volunteers finished the study, twenty-eight per group. Two volunteers of each group dropout the study. For the ECC group, the median dmfs index was 7.00 (Interquartile range: 10.00). The decomposed caries index showed $60 \%$ of white spot lesion, $20 \%$ of active cavitated lesion, $11 \%$ of filled without decay, $7 \%$ of inactive cavitated lesion, and $2 \%$ of filled with inactive caries lesion. The sex ratio $M-F$ of the sample was $1.15: 1.00$ for the CF group and 1.00:1.00 for the ECC group.

The effect of sucrose rinse, biofilm accumulation, and caries disease on the ionic concentration of calcium, phosphate, and fluoride is shown in Figure 1, Figure 2, and Figure 3 respectively. Detailed statistical information regarding simple effects is provided in Table 1. 
Table 1

Simple Effects of sucrose rinse, biofilm accumulation, and ECC on the ionic concentration of calcium, phosphate, and fluoride in saliva.

\begin{tabular}{|c|c|c|c|c|c|c|c|c|c|c|}
\hline \multirow[t]{2}{*}{ Simple Effects } & & \multicolumn{3}{|c|}{ Calcium } & \multicolumn{3}{|c|}{ Phosphate } & \multicolumn{3}{|c|}{ Fluoride } \\
\hline & & $a$ & $(\beta-1)$ & $\eta p^{2}$ & $a$ & $(\beta-1)$ & $\eta p^{2}$ & $a$ & $(\beta-1)$ & $\eta p^{2}$ \\
\hline \multirow{2}{*}{$\begin{array}{l}\text { Biofilm } \\
\text { accumulation } \\
\text { in CF children }\end{array}$} & Pre-rinse & 0.000 & 0.998 & 0.315 & 0.675 & 0.070 & 0.003 & 0.000 & 0.984 & 0.251 \\
\hline & Post-rinse & 0.000 & 0.991 & 0.265 & 0.362 & 0.147 & 0.015 & 0.464 & 0.112 & 0.010 \\
\hline \multirow{2}{*}{$\begin{array}{l}\text { Biofilm } \\
\text { accumulation } \\
\text { in ECC } \\
\text { children }\end{array}$} & Pre-rinse & 0.011 & 0.740 & 0.115 & 0.648 & 0.074 & 0.004 & 0.390 & 0.136 & 0.014 \\
\hline & Post-rinse & 0.017 & 0.677 & 0.101 & 0.585 & 0.084 & 0.006 & 0.072 & 0.438 & 0.061 \\
\hline \multirow[t]{2}{*}{$\begin{array}{l}\text { Rinse in CF } \\
\text { children }\end{array}$} & $\begin{array}{l}\text { No Biofilm } \\
\text { accumulation }\end{array}$ & 0.000 & 1.000 & 0.574 & 0.033 & 0.577 & 0.082 & 0.000 & 1.000 & 0.451 \\
\hline & $\begin{array}{l}\text { Biofilm } \\
\text { accumulation }\end{array}$ & 0.089 & 0.397 & 0.053 & 0.449 & 0.116 & 0.011 & 0.002 & 0.904 & 0.175 \\
\hline \multirow[t]{2}{*}{$\begin{array}{l}\text { Rinse in ECC } \\
\text { children }\end{array}$} & $\begin{array}{l}\text { No Biofilm } \\
\text { accumulation }\end{array}$ & 0.000 & 1.000 & 0.531 & 0.107 & 0.364 & 0.047 & 0.000 & 0.987 & 0.258 \\
\hline & $\begin{array}{l}\text { Biofilm } \\
\text { accumulation }\end{array}$ & 0.041 & 0.540 & 0.075 & 0.194 & 0.252 & 0.031 & 0.000 & 1.000 & 0.363 \\
\hline \multirow[t]{2}{*}{$\begin{array}{l}\text { ECC before } \\
\text { sucrose rinse }\end{array}$} & $\begin{array}{l}\text { No Biofilm } \\
\text { accumulation }\end{array}$ & 0.369 & 0.144 & 0.015 & 0.269 & 0.196 & 0.023 & 0.189 & 0.258 & 0.033 \\
\hline & $\begin{array}{l}\text { Biofilm } \\
\text { accumulation }\end{array}$ & 0.131 & 0.325 & 0.042 & 0.888 & 0.052 & 0.000 & 0.075 & 0.430 & 0.060 \\
\hline \multirow[t]{2}{*}{$\begin{array}{l}\text { ECC after } \\
\text { sucrose rinse }\end{array}$} & $\begin{array}{l}\text { No Biofilm } \\
\text { accumulation }\end{array}$ & 0.602 & 0.081 & 0.000 & 0.591 & 0.083 & 0.005 & 0.588 & 0.083 & 0.006 \\
\hline & $\begin{array}{l}\text { Biofilm } \\
\text { accumulation }\end{array}$ & 0.034 & 0.572 & 0.081 & 0.434 & 0.121 & 0.011 & 0.599 & 0.081 & 0.005 \\
\hline
\end{tabular}

As demonstrated in Figure 1, calcium concentration was significantly influenced by sucrose rinse, biofilm, and ECC. However, the absence of significant interaction between ECC and sucrose rinse, as well as between ECC and biofilm shows that ECC alone did not affect the calcium concentration in this experimental condition. More importantly, it was noticed that the combined effect of sucrose rinse and biofilm was the most significant condition to mobilize the calcium dynamic in the oral cavity, which can be demonstrated by the high effect size (type II error) and the strength (partial eta squared) of this interaction. Simple effects revealed that the calcium concentration was significantly lower in the biofilm accumulation situation as compared with the mechanical control of the biofilm situation, except for the CF children after sucrose rinse. In addition, it was evidenced that biofilm accumulation significantly increased the salivary calcium concentration in children with ECC after sucrose rinse whereas the mechanical control of biofilm reduced it in both groups. The strength of the mechanical control of biofilm can be demonstrated considering the high values of partial eta squared $\left(n \mathrm{p}^{2}\right.$ for $\mathrm{CF}=0.574$ and $\mathrm{np}^{2}$ for $\left.\mathrm{ECC}=0.531\right)$. Differences between $\mathrm{CF}$ children and children with ECC can be observed after sucrose rinse when the mechanical control of biofilm was absent. 
Concerning phosphate concentration (Figure 2), there was no significant interaction among sucrose rinse, biofilm, and caries disease. The main effects evidenced that biofilm accumulation influenced the phosphate concentration in saliva. Simple effects signals that this influence was significant for CF children in the mechanical control of biofilm situation. More specifically, a higher phosphate concentration in saliva was observed after sucrose rinse when CF children were submitted to mechanical control of biofilm.

Fluoride concentration was significantly altered by sucrose rinse, biofilm, and caries disease. Nevertheless, ECC alone did not influence the fluoride concentration when considering the within-subjects factors as biofilm accumulation and sucrose rinse. Of note, the interaction effect between sucrose rinse and biofilm seems to be more relevant. Simple effects show that biofilm accumulation reduced the fluoride bioavailability in the saliva of CF children. Sucrose rinse reduced the fluoride concentration in the mechanical control of biofilm situation as well as in the biofilm accumulation experimental condition in CF and ECC children. The strength of the combined effect of sucrose rinse and biofilm can be demonstrated considering the high proportion of variance explained by these experimental conditions (partial eta squared).

\section{Discussion}

ECC is the most important oral disease in early childhood, affecting the child's quality of life and promoting a potential source of cost to their parents and society (Tinanoff et al. 2019). As recently highlighted by the IAPD Bangkok Declaration (Pitts et al. 2019), the development of research regarding the prevention and comprehensive management of ECC should be the basis to improve the scope and the level of evidence in this field. In this context, this research was designed to improve the understanding of the two main factors implicated in the ECC physiopathology: sucrose exposure and biofilm accumulation. Here, it is important to emphasize that, the factors that rule the dysbiosis state in the oral environment can influence the onset of oral disease. Thus, the disease can be controlled along with childhood life. In such a scenario, host-related salivary electrolytes have important clinical implications in the maintenance of the mineral ultrastructure of the tooth surface.

The most intriguing question here is: Does the ECC as a chronic disease can influence these electrolytes and explain the boundaries of a healthy and sick oral ecosystem? In this sense, the recent study developed by Zhang et al. (2021) demonstrated that the assessment of children based only on the salivary biochemical electrolytes outperformed the prediction status of children with ECC when compared with classifiers based only on the salivary microbiome or based on both properties of saliva (microbial and biochemical electrolytes data). That is, the assessment of the caries prediction status in children was more reliable when it was based on the assessment of the inorganic composition of saliva (Zhang et al., 2021). Focusing on the bioavailability of the most important ions involved in the demineralization and remineralization events $\left(\mathrm{Ca}^{2+}, \mathrm{Pi}\right.$, and $\left.\mathrm{F}^{-}\right)$, our research brings some light and caveats to this question.

The study design included a simulation of a cariogenic challenge with a sugar concentration similar to sweetened foods and liquids commonly ingested by children and the simulation of proper oral hygiene supervised by an adult (situation in which there is biofilm control) and deficient oral hygiene (in which there is biofilm accumulation). To ensure the sample homogeneity and make the factors that directly influence caries disease more noticeable, some precautions were taken in the eligibility phase, such as the selection of preschool children with a well-defined age group (4 to 5 years) to avoid the effect of saliva and microbiome maturation, who had similar socioeconomic status, who had access to similar fluoride sources (1.100 ppm fluoride toothpaste and $0.7 \mathrm{ppm}$ fluoridated water) and who shared the same 5 meals a day. 
Interestingly, the frequency of sucrose consumption was around 5 times a day for CF children as well as for children with ECC (results not shown), which is considered a sugar ingestion of risk for ECC (Folayan et al. 2020, Parisotto et al. 2015). In line with this assumption, in the group of children with ECC, it was observed that $80 \%$ of caries lesions were in the active situation (60\% active white spot lesions and $20 \%$ active cavitated lesions). This critical situation in individuals having early childhood caries inspired us to investigate the salivary factors associated with the physiopathology of dental caries in this group of children and to provide meaningful information to trigger more specific and efficient diagnosis, prevention, and care for this disease.

This study was the first to demonstrate that the concentrations of calcium and fluoride in the saliva of preschool children were significantly influenced by transient exposure to sucrose (rinse) and by biofilm accumulation. Moreover, the high values of partial eta squared in these interactions can evidence the strength of this combined effect (see partial eta squared in Figure 1 and Figure 3). On the other hand, in the children who experienced ECC, the calcium and fluoride concentrations did not behave differently from CF children in the experimental conditions of the study. This result strengthens the findings provided by de Sousa et al. (2020) who demonstrated that the status of the disease (presence or absence) did not influence the bioavailability of naturally occurring salivary electrolytes such as calcium and fluoride. In addition, these results, add more information to the bioavailability of these ions in the saliva medium. Thus, this emerging body of knowledge can be relevant for future experimental designs about ECC physiopathology, which must warrant that the combination of proximal factors (simple sugars intake and oral hygiene) should be considered or isolated to explain what changes in the oral environment could be expected in each situation and how these changes could be controlled to avoid more aggressive forms of ECC.

Calcium concentration was significantly lower in the biofilm accumulation situation as compared with the mechanical control of the biofilm situation in CF children as well as in children with ECC. In the biofilm accumulation situation, these results can be explained by the metabolic profile (more saccharolytic) of the oral environment, which is capable to promote an important variation on salivary parameters such as the salivary flow rate, $\mathrm{pH}$, and buffering capacity (Lima-Holanda et al., 2021). Thus, the frequent $\mathrm{pH}$ fall may disrupt the equilibrium of calcium ions on the enamel surface and provide a coercive scenario for uptake of calcium ions from saliva. Moreover, there is a possibility that salivary calcium can be trapped by acids, mainly lactate (Astasov-Frauenhoffer et al., 2017), reducing its saliva bioavailability.

There was a tendency to an increase in the salivary calcium after sucrose rinse in the biofilm accumulation situation, whereas a decrease in this ion concentration was noticed in mechanical control of the biofilm situation. As an exception, it was observed that sucrose rinse did not modify the calcium concentration in saliva of CF children in the biofilm accumulation situation. This could be interpreted with caution since the alpha value was in the margin of significance $(p=0.089)$ and the effect size (type II error) and the strength (partial eta squared) were low. The opposite tendency for calcium concentration in biofilm accumulation and mechanical control of biofilm situation can be a result of a more organized biofilm with an extracellular polymeric matrix capable to control the ionic and molecular transport across the biofilm structure (Hwang et al., 2016; Xiao et al., 2017). As demonstrated by AstasovFrauenhoffer et al. (2017), cariogenic bacteria species have a regulatory mechanism to eliminate possibly toxic amounts of calcium ions, binding to them to strengthen their advanced biofilm nuclei, and at the same time, affecting the free calcium ratios for remineralization that would be available in the biofilm fluid. In other words, the less organized biofilm structure provides a more meaningful calcium uptake from saliva to the tooth-enamel interface, reducing its bioavailability in saliva, whereas a more organized biofilm does not. Thus, as the organized biofilm can be a barrier to ionic transport, the effect of masticatory and gustatory stimulus in the increase in the calcium concentration can be more evident, especially in the group of children with ECC. 
When the observed ion is the inorganic phosphate, the simple effects showed that there was a medium and significant effect of the sucrose rinse on the CF children in the situation of mechanical control of the biofilm. Considering the findings of Sousa et al. 2020, which have important methodological similarities with our study, CF children maintain their inorganic phosphate concentration stable after the cariogenic challenge with sucrose rinse. $A$ deeper theoretical consideration can be inferred from these contrasting data because in CF children, they reinforce that the mechanical control of the biofilm rules the response to the transient exposure to sucrose more than ECC alone. In this context, it should be pointed out that only biofilm accumulation influenced the phosphate concentration in saliva in the experimental conditions of our study. On the other hand, previous clinical studies demonstrated that phosphate concentration is inversely related to the dental caries experience (Shaw et al., 1983; Sigh et al., 2015). The contrasting results may well be explained considering the differences in the design of the various studies. In the study of Shaw et al., (1983), the authors used unstimulated submandibular and sublingual saliva samples obtained from adolescents aged 13-15 years old, who had a mean DMFS of 25.9 (SD \pm 8.5$)$. In the investigation performed by Sigh et al., (2015), the experimental group was composed of 4 to 8 years old caries active children who presented a DMFS $\geq 5$. Furthermore, it should be perceived that in none of these referred studies the individuals were exposed to a cariogenic challenge and/or refrained from controlling their biofilm.

As expected, in both groups, the sucrose rinse reduced the fluoride concentration in the mechanical control of biofilm and in situations of the biofilm accumulation. Thus, our research reinforces the overwhelmed influence of sucrose rinse and biofilm on the behavior of this ion when compared with the caries experience. However, it should be noticed that although the interaction between sucrose rinse and disease was not significant, it is important to consider that the $p$-value of the interaction between the two variables was 0.07 with low power but medium partial eta squared (see Figure 3). This data can inform that maybe the difference exists but the sample size did not allow demonstrating this effect. More specifically, biofilm accumulation caused an impact on the fluoride bioavailability in the pre-rinse situation in CF children $\left(\alpha=0.000 ; \beta-1=0.984 ; \eta p^{2}=0.251\right)$ and probably in the post-rinse situation in ECC children $\left(a=0.072 ; \beta-1=0.438 ; n p^{2}=0.061\right)$, if the power of the analysis increase. Here, the number of participants inputs some restrictions for the statistical inference and design. In this respect, we argue that the three-way analysis of variance has a complex statistical structure and limited studies are available with similar factors investigated together to assess the expected variability and appropriate sample size.

Fluoride concentrations in saliva may be related to fluoride concentrations in the biofilm/tooth interface, which is the site of action for the fluoride effect on the caries control. There is a consensus that biofilm gradually concentrates the fluoride available from saliva, enabling higher levels of $\mathrm{F}^{-}$ions available on the tooth surface (Featherstone, 1999), which can explain as why the presence of biofilm causes a reduction in the levels of available fluoride in saliva in CF children. However, the higher levels of salivary fluoride in CF children when the mechanical control of biofilm was performed, could represent a state of more stability against tooth demineralization since more ionic components would be available for crossing the disorganized biofilm structure and would reduce the chemical aggression promoted by the cariogenic challenge. Thus, biochemical processes in the oral cavity can increase the environmental electrolytes supersaturation around and within the tooth surface biofilm structure (Hara and Zero, 2010), which can be highly indicative of an oral health status.

Several strengths can be pointed out in this research, such as the careful selection of an adequate and specific sample, the collection of data simulating usual day-to-day conditions, and the statistical design with a robust and extremely conservative rank test of interaction. However, some limitations should be displayed. Firstly, we only analyzed saliva samples and did not verify the bioavailability and behavior of the inorganic composition in biofilm samples, as it could provide us with important results. Secondly, there are no longitudinal data to provide 
information on how the bioavailability of ions in saliva changes across time, considering the outcome ECC. This limitation includes the knowledge regarding the boundaries between children with ECC who control or maintain the disease activity and CF children who develop ECC or maintain their health status. Thus, future investigation considering a longitudinal design is highly recommended to address these aspects of ECC. Thirdly, the design of this research considered the caries experience and activity as a parameter for classifying children with ECC. In this sense, the absence of stratification of ECC components in an experimental design can provide a potential source of bias.

In conclusion, this study demonstrated that the combined effect of biofilm accumulation and sucrose rinse modifies the bioavailability of calcium and fluoride in the saliva of caries-free children and children with ECC. On the other hand, for the phosphate concentration, only the sucrose rinse seems to be relevant for caries-free children in the absence of biofilm accumulation.

\section{Material And Methods}

\section{Research Subjects}

The sample size was calculated using the Gpower 3.1 software, assuming a difference between two independent means. The calculation was performed considering an a value of $0.05, \beta$ value of 0.15 , allocation rate of $1 / 1$, and confidence interval of 0.95 . The sample size was estimated according to the mean and standard deviation of the salivary calcium concentration in the saliva of children with ECC and caries-free children (Hegde et al., 2014), resulting in 25 subjects for each group. The calculated number (25) was increased to 30 to compensate for possible dropouts by the subject during the experiment. Thus, 30 preschoolers per group were included in this study.

The sampling was of the probabilistic type by conglomerates. Once the clusters were selected (public daycare centers in the city of Piracicaba, São Paulo, Brazil), all children were analyzed and included in the study according to the eligibility criteria. Children with caries lesions $(\mathrm{dmfs} \geq 1)$ and caries-free ( $\mathrm{dmfs}=0)$, aged 3 to 4 years (at the beginning of the study) and of both sexes were included in the study. Preschool children whose parents or guardians refused to participate in the research or who did not cooperate with the clinical examinations were excluded from the research, as well as those who, in the clinical examination, presented periodontal disease (assessed using the Gingival Bleeding Index (World Health Organization, 2013), severe fluorosis or who were using any orthodontic appliance. In addition, children who had any systemic disease, neuromotor or communication difficulties, and who were under antibiotic therapy were excluded. This information was accessed through a questionnaire sent to parents.

\section{Experimental Design}

A quasi-experimental, parallel, study was carried out in a sample consisting of children with ECC and caries-free children, to investigate the influence of two experimental conditions on the inorganic composition of saliva: biofilm accumulation and cariogenic challenge with a $20 \%$ sucrose solution(de Sousa et al., 2020).

Adequate oral hygiene (situation of biofilm control) and poor oral hygiene (no biofilm control) was performed at different times with an interval of at least one week. These interventions were conducted as follows (Figure 4):

- Absence of biofilm accumulation: for two days, the volunteers were submitted to mechanical control at least three times a day with a technique suitable for the preschool age group (Fones technique). Tooth brushing was performed 1 hour before saliva collection and always supervised by the researcher in the morning (between 8 am and 9 am) 
and in the afternoon (between 4 am and $5 \mathrm{am}$ ). Phone calls and/or text messages were used as a strategy to remind parents about nighttime toothbrushing.

- Biofilm accumulation: for two days the parents were instructed, through phone calls and/or text messages, to abstain from brushing their children's teeth. To strengthen this recommendation, the children were also instructed about the procedure. Visualization of the visible biofilm on the upper incisors (Alaluusua and Malmivirta, 1994) was used as a control for abstaining from brushing.

In each situation, samples of stimulated saliva were collected before rinse for 1 minute with $10 \mathrm{~mL}$ of a $20 \%$ sucrose solution and after five minutes. The saliva samples were used to determine the inorganic composition of $\mathrm{Ca}^{2+}, \mathrm{Pi}$, and $\mathrm{F}^{-}$.

\section{Clinical Examination}

The clinical examination was performed at the daycare center, by a single previously calibrated dentist. The examiner was trained in a population of the same age as the sample. The training exercise consisted of two steps (theoretical and clinical). The first stage involved a theoretical discussion of the World Health Organization (WHO) caries diagnostic criteria, plus the criteria for the diagnosis of active caries lesions (Nyvad et al., 1999). The WHO and active caries lesions criteria and codes were adopted following the protocol of Assaf et al., (2006). The second step was carried out to assess the consistency of the clinical analysis. The examiner and a calibrated pediatric dentist (gold standard) evaluated the indices in a sample of 10 children, selected at random. The inter-examiner reliability was verified using the Kappa statistic $(\mathrm{K}=0.89)$ and the reproducibility of the diagnosis was determined by the intra-examiner Kappa index based on reassessments performed in $60 \%$ of the children after a one-week interval $(\kappa=0.95)$.

During the examination, individual protective equipment was used, as well as sterilized and individual clinical material for each child. For the examination, a portable flashlight with LED light (Sf15540, Wat Nichia Philips, Brazil), a clinical mirror, and a blunt tip explorer (Indusbello, Londrina-PR, Brazil) and/or sterilized gauzes (Medi House, São Paulo-SP, Brazil) were used. Portable dental equipment with a triple syringe (Odontocase Basic Line, Rio de Janeiro, Brazil) was used to facilitate the visualization and the correct diagnosis of the initial caries lesions (active white spot lesion).

Children who needed dental treatment were referred to the Pediatric dentistry clinic of the Piracicaba Dental School UNICAMP, where they received comprehensive care.

\section{Saliva Collection}

Stimulated saliva collection was performed with a piece of Parafilm® M (Pechiney Plastic Packaging, Inc. Manuf. and Markets Plastic, Chicago, Illinois, USA). The saliva produced in the initial 30 seconds was discarded and the remaining saliva was collected for 5 minutes in autoclaved graduated tubes. After collection, the saliva samples were centrifuged at $16,097.2 \mathrm{~g}$ for $15 \mathrm{~min}$ and then transferred for $2.0 \mathrm{~mL}$ eppendorf identified microtubes, and kept frozen at $-40^{\circ} \mathrm{C}$ until the analyses were performed.

\section{Calcium analysis}

Saliva calcium concentration was analyzed by the direct colorimetric method using a microplate spectrophotometer (PowerWave HT, BioTek Instruments, Winooski, VT, USA). Briefly, $25 \mu \mathrm{L}$ of saliva were pipetted into a 96-well plate to react with $125 \mu \mathrm{L}$ of calcium-sensitive reagent (Arsenazo III). The reader was precalibrated against a calcium 
carbonate standard curve (0-100 $\mu \mathrm{g} / \mathrm{mL})$ and readings were performed using an $\lambda 650 \mathrm{~nm}$ of absorbance (Brown and Rydqvist, 1981). The ionic concentration of calcium in saliva was obtained from the absorbance values using a linear equation $(y=a x+b)$ in a curve fit above 0.98. Samples were analyzed in duplicate. Calcium concentrations were expressed as $\mu \mathrm{g} / \mathrm{mL}$.

\section{Phosphate Analysis}

The phosphate concentration in saliva was analyzed using the colorimetric method using a microplate spectrophotometer (PowerWave HT, BioTek Instruments, Winooski, VT, USA). Briefly, $25 \mu \mathrm{L}$ of saliva were pipetted into a 24-well plate to react with the phosphate reducing agents (molybdic acid and alpha-aminonaphthol sulfonic acid). The reader was previously calibrated with a phosphate standard curve $(0-8.27 \mu \mathrm{g} / \mathrm{mL})$. Readings were performed using the absorbance of $\lambda 660 \mathrm{~nm}$ (Chen et al., 1956). Ionic concentration of phosphate was obtained from the absorbance values using a linear equation $(y=a x+b)$ in a curve fit above 0.99 . Samples were analyzed in duplicate. Phosphate concentrations were expressed as $\mu \mathrm{g} / \mathrm{mL}$.

\section{Fluoride Analysis}

The fluoride concentration in saliva was measured by the direct method (Martinez-Mier et al., 2011). A specific electrode for fluoride ion (BN Model 9409, Orion, Cambridge, MA, USA) and a potentiometer (Model 720 Orion, Cambridge, MA, USA) were used for fluoride analysis and each sample was analyzed in duplicate. Before the readings, calibration curves were obtained with fluoride standards ranging from 0.01 to $0.1 \mathrm{mg} \mathrm{F} / \mathrm{mL}$ and TISAB III (1:10) (Thermo Electron, Fisher Scientific, Walthamam, MA, USA).

Results expressed in $\mathrm{mV}$ by the potentiometer were converted to concentration of fluoride ion using a standard correlation curve $\left(r^{2}>0.99\right)$ based on the linear regression of the calibration curve. Fluoride concentrations were expressed as $\mu \mathrm{g} / \mathrm{mL}$.

\section{Statistical Approach}

The SPSS software for Windows, version 21.0 (SPSS, Inc., Chicago, IL, USA) and the GraphPad Prism 7.04 software (GraphPad Software, La Jolla, USA) were used for statistical inferences.

The normality of data variances was checked using the Shapiro-Wilk test. Data of calcium, phosphate, and fluoride followed the Gaussian distribution and the equality of variances. Data of caries index was represented as median and interquartile range due to non-Gaussian distribution.

The three-way mixed model of analysis of variance was used to determine the effect of biofilm accumulation and sucrose rinse (within subjects' effects) and early childhood caries (between subjects' effects) on the test variables (dependent variables: $\mathrm{Ca}^{2+}, \mathrm{Pi}$, and $\mathrm{F}^{-}$). The Box's $\mathrm{M}$ test was used to prove the equality of multiple variancecovariance matrices considering the 0.001 significance level. Significant interaction in analysis of variance ruled the need for simple effects tests to reveal the degree to which one factor is different from other factors, considering the Bonferroni adjustment to avoid familywise error. The level of significance established for the analysis was 0.05 , considering the two-tailed test hypothesis.

\section{Declarations}

\section{Ethics declarations}


Approval for human experiments. Methods were carried out following the Helsinki declaration and the regulatory guidelines and norms obey the 466/12 resolution for research ethics in Brazil. The Research Ethics Committee of the Piracicaba Dental School University of Campinas approved this research. (CAAE: 70777517.9.0000.5418). Parents or guardians who agreed with the inclusion of children in the research, singed an informed consent form (FICF), authorizing their participation in the research.

\section{Acknowledgments}

We especially thank the volunteers for their participation in this study, which was based on a dissertation submitted by the first author to Piracicaba Dental School, University of Campinas, in partial fulfillment of the requirements for a master's degree in Dentistry (Pediatric Dentistry Area). This study was supportedby the São Paulo Research Foundation-FAPESP (Grant No 2017/17630-8).

\section{Author Contribution Statement}

C. Lopes Crescente participated in the design and conception of the study, data acquisition, interpretation of results, writing, and critical review of the manuscript; A. T. Lima-Holanda participated in the data acquisition, interpretation of results, writing, and critical review of the manuscript; E. T. Sousa participated in the data acquisition, interpretation of results, writing, and critical review of the manuscript; C. Steiner-Oliveira participated in the design and conception of the study, data acquisition, interpretation of results, writing, and critical review of the manuscript; M. Nobre-dosSantos participated in the design and conception of the study, data acquisition, interpretation of results, writing, and critical review of the manuscript. All authors agreed with the final version of the paper.

\section{Conflict of Interest}

The authors have no competing interests as defined by Nature Research, or other interests that might be perceived to influence the results and/or discussion reported in this paper.

\section{Funding}

The workwassupportedby Fundação de Amparo à Pesquisa do Estado de São Paulo (FAPESP) [Grant. No. 2017/17630-8] andFundação de Desenvolvimento da Unicamp (FUNCAMP) [Grant. No.45631-18].

\section{References}

1. Tinanoff N, et al. Early childhood caries epidemiology, aetiology, risk assessment, societal burden, management, education, and policy: Global perspective. Int J Paediatr Dent. 29(3), 238-48, https://doi.org/10.1111/ipd.12484 (2019).

2. Koo, H., Falsetta, M. L. \& Klein, M. I. The exopolysaccharide matrix: a virulence determinant of cariogenic biofilm. Dent. Res. 92, 1065-1073, https://doi.org/10.1177/0022034513504218 (2013)

3. Marsh, P. D. In sickness and in health-what does the oral microbiome mean to us? An ecological perspective. Dent. Res. 29, 60-65, https://doi.org/10.1177/0022034517735295 (2018).

4. Zaura, E. et al. On the ecosystemic network of saliva in healthy young adults. ISME J. 11, 1218-1231. https://doi.org/10.1038/ismej.2016.199 (2017).

5. Featherstone, J. D. The continuum of dental caries - evidence for a dynamic disease process. Dent. Res. 83, C39-C42, https://doi.org/10.1016/j.jdent.2010.04.003 (2004). 
6. Pappa E, Kousvelari E, Vastardis H. Saliva in the "Omics" era: A promising tool in paediatrics. Oral Dis. 25(1), 1625, https://doi.org/10.1111/odi.12886 (2019).

7. Lenander-Lumikari, M. \&Loimaranta, V. Saliva and dental caries. Dent. Res. 14, 40-47, https://doi.org/10.1177/08959374000140010601 (2000).

8. Jain, S. et al. Effect of diet modification on salivary parameters and Oratest in high-caries-risk individuals. J. Clin. Pediatr. Dent.11, 34-39, https://doi.org/10.5005/jp-journals-10005-1480 (2018).

9. Garcla-Godoy, F. \& Hicks, M. J. Maintaining the integrity of the enamel surface: the role of dental biofilm, saliva and preventive agents in enamel demineralization and remineralization. Am. Dent. Assoc. 139, 25S-34S (2008).

10. Dawes, C. Salivary flow patterns and the health of hard and soft oral tissues. JADA 139, 18S-24S, https://doi.org/10.14219/jada.archive.2008.0351 (2008).

11. de Sousa, E. T. et al. Changes in the salivary electrolytic dynamic after sucrose exposure in children with Early Childhood Caries. Rep. 10, 4146. https://doi.org/10.1038/s41598-020-61128-6 (2020).

12. Lima-Holanda AT, de Sousa ET, Nobre-Dos-Santos M, Steiner-Oliveira C. The role of mechanical control of biofilm in the salivary pH after sucrose exposure in children with early childhood caries. Sci Rep. 11(1), 7496, https://doi.org/10.1038/s41598-021-86861-4 (2021).

13. Pitts NB, Baez RJ, Diaz-Guillory C, et al. Early Childhood Caries: IAPD Bangkok Declaration. J Dent Child (Chic). 86(2), 72, (2019).

14. Zhang Y, Huang S, Jia S, et al. The predictive power of saliva electrolytes exceeds that of saliva microbiomes in diagnosing early childhood caries. J Oral Microbiol. 13(1), 1921486, https://doi.org/10.1080/20002297.2021.1921486 (2021).

15. Folayan $\mathrm{MO}$ et al. Early childhood caries and its associations with sugar consumption, overweight and exclusive breastfeeding in low, middle and high-income countries: an ecological study. PeerJ. 8:e9413, (2020).

16. Parisotto TM et al. Can insoluble polysaccharide concentration in dental plaque, sugar exposure and cariogenic microorganisms predict early childhood caries? A follow-up study. Arch Oral Biol. 60(8), 1091-1097, https://doi.org/10.1016/j.archoralbio.2015.04.003 (2015).

17. Astasov-Frauenhoffer, M. et al. Exopolysaccharides regulate calcium flow in cariogenic biofilms. 12, e0186256, https://doi.org/10.1371/journal.pone.0186256 (2017).

18. Hwang, G. et al. Simultaneous spatiotemporal mapping of in situ pH and bacterial activity within an intact 3D microcolony structure. Rep. 6, 32841, https://doi.org/10.1038/srep32841 (2016).

19. Xiao, J. et al. Biofilm three-dimensional architecture influences in situ pH distribution pattern on the human enamel surface. J. Oral Sci. 9, 74-79. https://doi.org/10.1038/ijos.2017.8(2017).

20. Shaw L, Murray JJ, Burchell CK. Calcium and phosphorous content of plaque and saliva in relation to dental caries. Caries Res. 17, 543e548, (1983).

21. Singh, S. et al. A Saliva as a prediction tool for dental caries: An in vivo J. Oral. Biol. Craniofacial Res. 5, 59-64, https://doi.org/10.1016/j.jobcr.2015.05.001 (2015).

22. Featherstone JD. Prevention and reversal of dental caries: role of low-level fluoride. Dent Oral Epidemiol. 27(1), 31-40, https://doi.org/10.1111/j.1600-0528.1999.tb01989.x 1999.

23. Hara AT and Zero DT. The caries environment: saliva, pellicle, diet, and hard tissue ultrastructure. Dent Clin North Am. 54(3), 455-467, https://doi.org/10.1016/j.cden.2010.03.008 (2010).

24. Hegde, A. M., Naik, N. \& Kumari, S. Comparison of salivary calcium, phosphate and alkaline phosphatase levels in children with early childhood caries after administration of milk, cheese, and GC tooth mousse: An in vivo J.

Page $12 / 15$ 
Clin. Pediatr. Dent. 38, 318-325 (2014).

25. World Health Organization. Oral health surveys: basic methods - 5th edition. (Geneva, 2013).

26. Alaluusua, S. \&Malmivirta, R. Early plaque accumulation-a sign for caries risk in young children. Community Dent. Oral. Epidemiol. 22, 273-276, https://doi.org/10.1111/j.1600-0528.1994.tb02049.x (1994).

27. Nyvad, B. et al. Reliability of a new caries diagnostic system differentiating between active and inactive caries lesions. Caries Res. 33, 252-260, https://doi.org/10.1159/000016526 (1999).

28. Assaf, A. V. et al. Effect of different diagnostic thresholds on dental caries calibration - a 12-month evaluation. Community Dent. Oral. Epidemiol. 34, 213-219, https://doi.org/10.1111/j.1600-0528.2006.00278.x (2006).

29. Brown, H. M. \&Rydqvist, Bo. Effect of $\mathrm{pH}$, ionic strength, and Arsenazo III concentration on equilibrium binding evaluated with Ca2+ ion-sensitive electrodes and absorbance measurements. J. 36, 117-137, https://doi.org/10.1016/S0006-3495(81)84720-0 (1981).

30. Chen, P. S., Toribara, T. Y. \& Warner, H. Micro determination of phosphorus. Chem. 28, 1756-1758 (1956).

31. Martinez-Mier, E. A. et al. Development of gold standard ion-selective electrode-based methods for fluoride analysis. Caries Res. 45, 3-12; https://doi.org/10.1159/000321657 (2011).

\section{Figures}

CF $\quad$ ECC

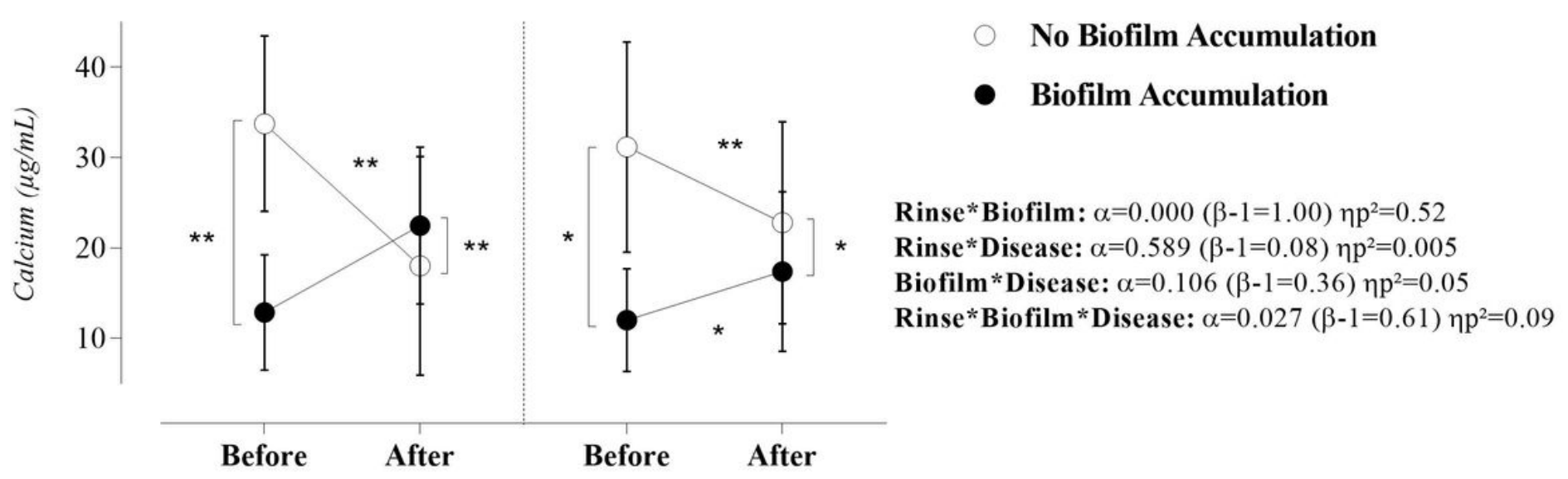

Figure 1

Effect of sucrose rinse, biofilm accumulation, and ECC on the ionic concentration of calcium.

Footnote: Statistical analysis was performed with a sample of 56 volunteers, 28 per group. $\mathrm{np}^{2}$ : Partial eta squared. Data were plotted as means and standard deviations. When significant interactions were found, the main effects were suppressed. A single asterisk represents a significant $p$-value $\leq 0.05$. A double asterisk represents a $p$-value $\leq$ 0.01 . 


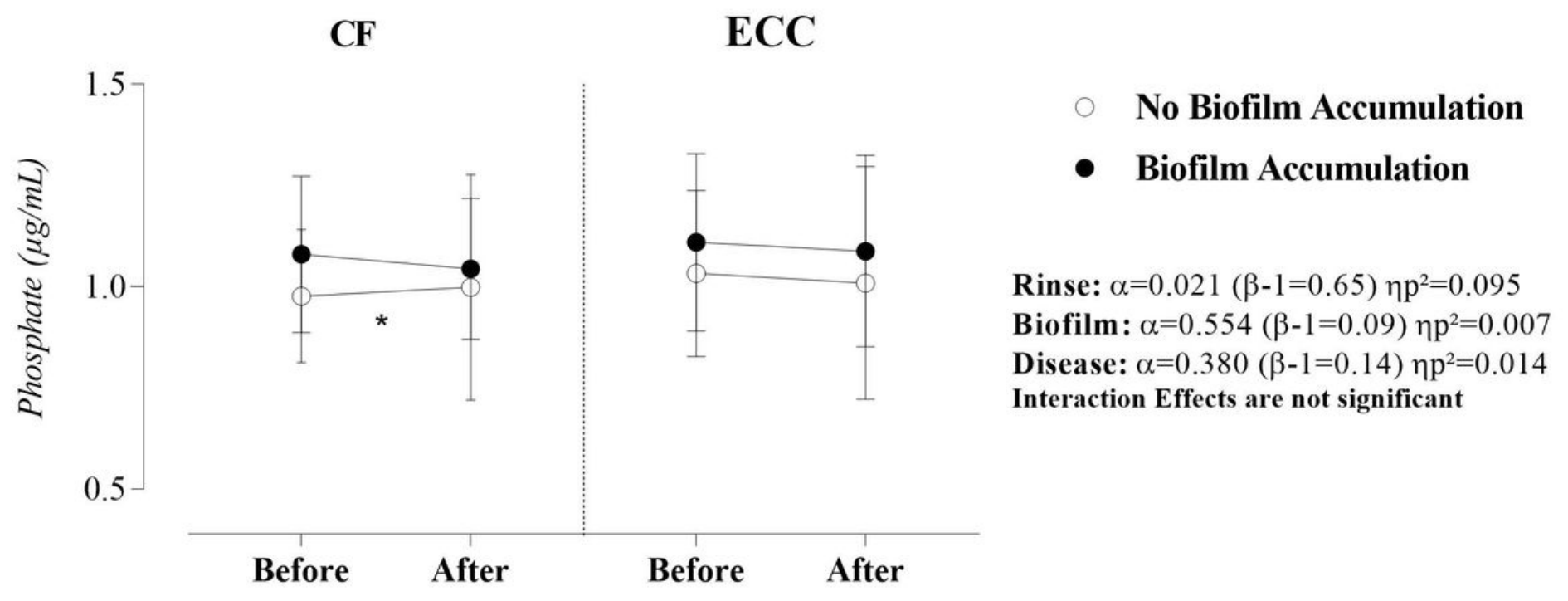

Figure 2

Effect of sucroserinse, biofilm accumulation, and ECC on the ionic concentration of inorganic phosphate.

Footnote: Statistical analysis was performed with a sample of 56 volunteers, 28 per group. $\eta p^{2}$ : Partial eta squared. Data were plotted as means and standard deviations. When significant interactions were found, the main effects were suppressed. A single asterisk represents a significant $p$-value $\leq 0.05$. A double asterisk represents a $p$-value $\leq$ 0.01 .

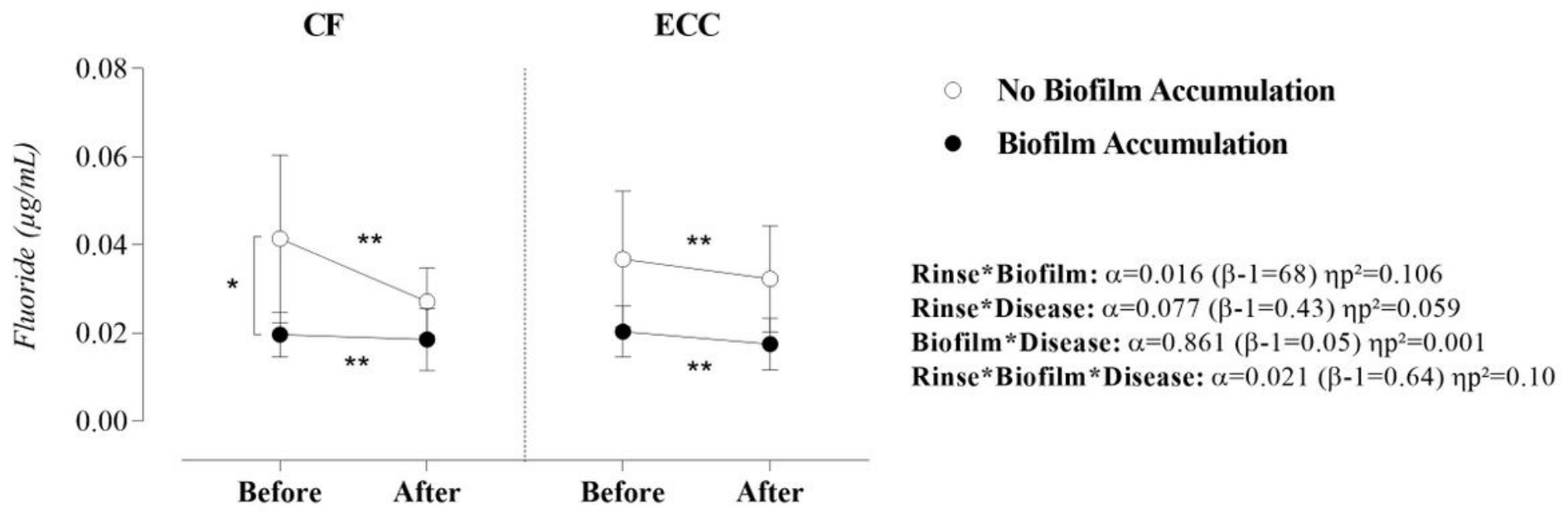

Figure 3

Effect of sucroserinse, biofilm accumulation, and ECC on the ionic concentration of fluoride.

Footnote: Statistical analysis was performed with a sample of 56 volunteers, 28 per group. $\eta p^{2}$ : Partial eta squared. Data were plotted as means and standard deviations. When significant interactions were found, the main effects were suppressed. A single asterisk represents a significant $p$-value $\leq 0.05$. A double asterisk represents a $p$-value $\leq$ 0.01 . 


\section{Clinical Phase}

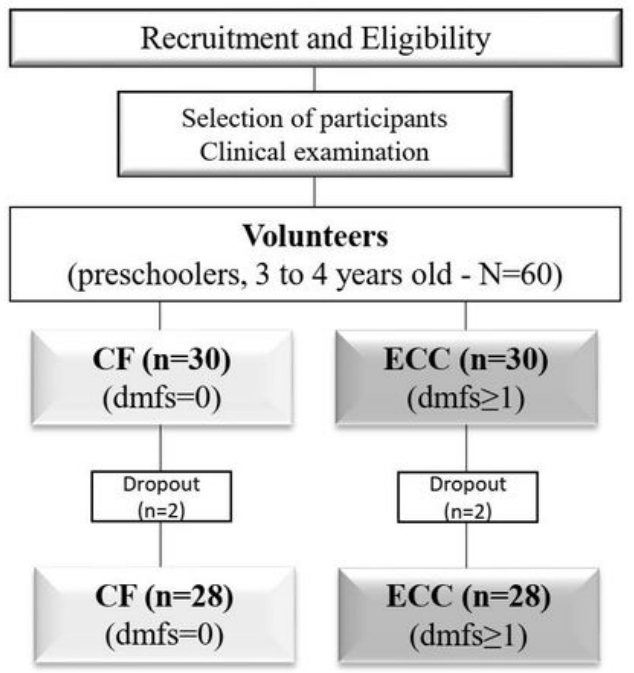

\section{Experimental Phase}

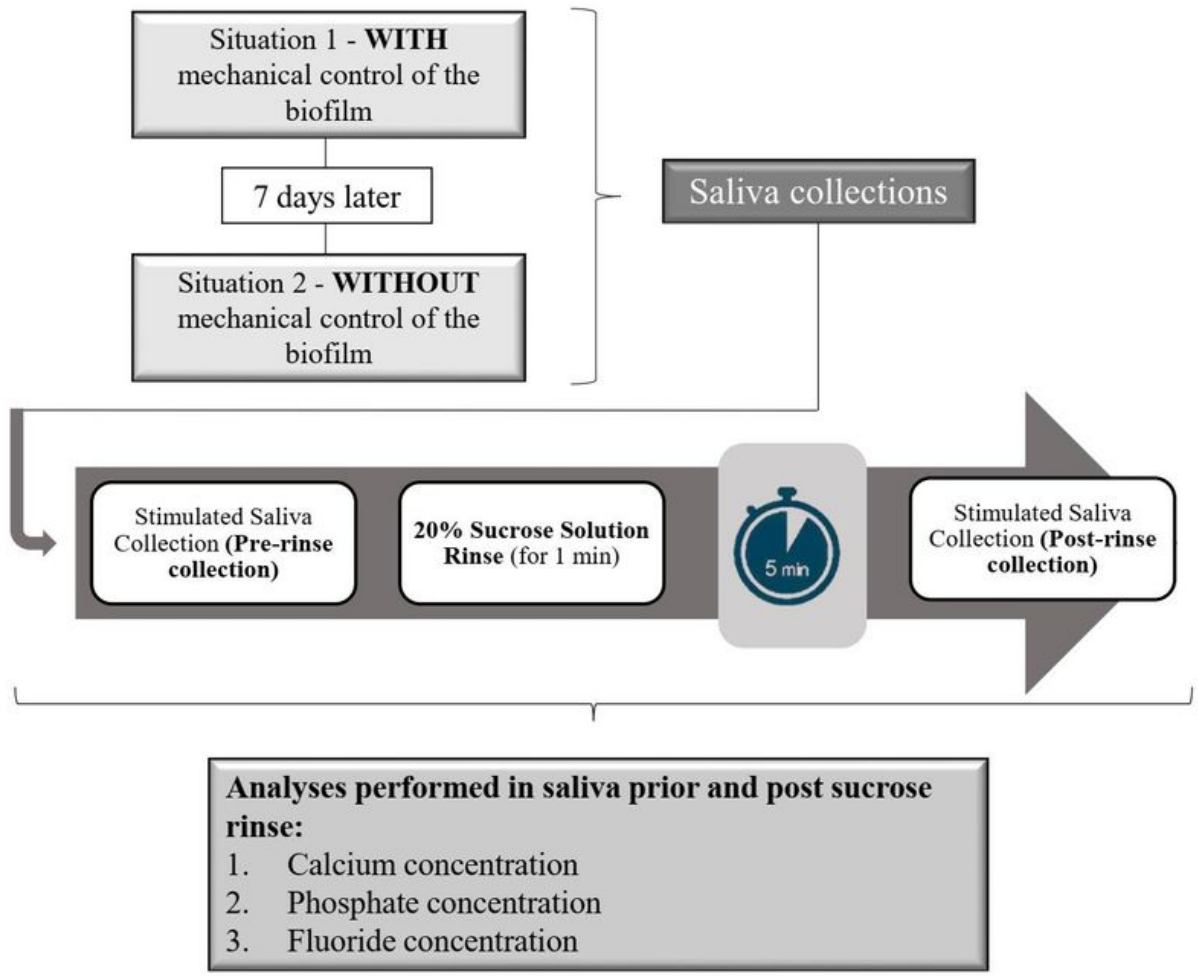

Figure 4

Flowchart of the Research Experimental Design. 